\title{
Locally advanced angiocentric nk/t cell lymphoma of nasal cavity
}

\begin{abstract}
Background: Angio centric NK/T-cell lymphoma represents a rare malignant entity, characterized by progressive and relentless destruction of the affected tissues. Typically, this type of lymphoma originates in the nasal cavity, the palate or midfacial region characterized by vascular invasion and unrelenting destruction of the midline structures of the palatine structures and nasal fossa causing severe pain. EpsteinBarr virus (EBV) infection has important pathogenetic role as it has been isolated in practically all cases of angiocentric NK/T-cell lymphoma. The prognosis of locally advanced cases remains very poor despite treatment.
\end{abstract}

Case presentation: We are reporting a case of a 45 year young female with an advanced angiocentric NK/T-cell lymphoma who had progression to deep necrotic ulcer in the midline of palate destroying palatal tissues thus creating an oronasal fistula complicated with secondary infection. Patient received standard CHOP regimen with high palliative external beam radiotherapy followed by metronomic chemotherapy with lenalidomide and have considerable regression of localized disease and got superb palliation.

Conclusion: Combination of locoregional radiotherapy and CHOP chemotherapy are the mainstay of treatment till now. Prosthetic treatment of possible oronasal fistulas/defects can significantly improve the patient's quality of life. Present case highlights the important role of local radiotherapy and metronomic chemotherapy with lenalidomide for symptomatic improvement.

Keywords: angiocentric NK/t-cell lymphoma, chop chemotherapy, palliative radiotherapy, metronomic chemotherapy with lenalidomide
Volume 9 Issue 6 - 2018

Anil Kumar Dhull, Parul Gupta, Vivek Kaushal

Department of Radiation Oncology, Post Graduate Institute of Medical Sciences, India

Correspondence: Anil Kumar Dhull, Radiation Oncologist, Department of Radiation Oncology, Post Graduate Institute of Medical Sciences, PO Box 100, GPO Rohtak-12400I, Haryana, India, Fax+9| | 262299999, Email anilkbhull@gmail.com

Received: October 20, 2018 | Published: November 02, 2018
Abbreviations: ENKTCL, extra nodal angiocentric nk/t-cell lymphoma; CHOP, cyclophosphamide, doxorubicin, vincristine, prednisone; NHLs, non-hodgkin's lymphomas, NK, natural killer; CT-PNS,computed tomography paranasal sinuses CK-cytokeratin; EBRT, external beam radiotherapy; PET-CT, positron emission tomography-computerized tomography; 18-FDG PET-CT-FDG, avid positron emission tomography computerized tomography; DNA- Deoxyribonucleic acid; EBV, epstein-barr virus; ISH, Insitu hybridization; EBER, sepstein-barr encoding region; CT, computerized tomography; MRI, magnetic resonance imaging; WG, wegener's granulomatosis; HSCT, hematopoietic stem cell transplantation

\section{Introduction}

Angiocentric NK/T-cell lymphoma also known as extranodal nasal-type NK/T-cell lymphoma and midline lethal granuloma is a rare lymphoid malignancy with rapid progression. Developed countries have low prevalence i.e. $0.4-2.2 \%$ among all non-Hodgkin's lymphomas (NHLs) contrary to Asia and Central \& South America where it is $6-7 \%$ of all NHLs. ${ }^{1}$ It is a highly aggressive tumor with poor prognosis and a median survival time of $<12$ months and a previous five-year survival rate of $30-40 \%$. However, this five-year survival rate has increased to $71 \%$ in recent years with the application of intensive treatment methods. ${ }^{2}$ The aim of this report is to present a case of locally advanced angiocentric NK/T-cell lymphoma of nasal cavity invading hard palate, emphasizing its clinical and histopathological differential diagnosis and treatment.

\section{Case summary}

A 45year old female with no prior co morbid conditions presented with history of nasal obstruction for two and a half years, associated with severe crusting which was relieved by alkaline nasal rinses and painless ulceration of soft palate for 5 months which gradually progressed towards hard palate to form an or nasal fistula, associated with foul smelling discharge, halitosis, distortion of nasal bridge and swelling over right side face. There was no swallowing difficulty, change in voice quality, fever or lymphadenopathy. On examination, an irregular lesion of $5 \times 6 \mathrm{~cm}$ with foul smelling discharge and necrotic debris without inflammatory signs was present on hard palate with mild crusting of nasal cavity. All hematological and biochemical parameters were within normal limits. Computed Tomography paranasal sinuses (CT-PNS) revealed absence of antero inferior nasal septum with defect in hard palate with or nasal communication. Histopathological examination after palatal biopsy and nasal biopsy revealed stratified squamous mucosa with underlying soft tissue infiltration by lymphoid cells in palatal lesion and ulcerated squamous epithelium, sub mucosal tissue and sero mucosal glands with large areas of necrosis and infiltration by lymphoid (small dark blue) cells in nasal lesion. The neoplastic cells illustrated wide spread positivity for CCA, CD3 and CD5 immunocyto chemical stain while stain for cytokeratin (CK) were negative. The histopathological appearance and immuno histo chemical profile confirmed it to be angiocentric T cell lymphoma. Clinically the patient was labelled as locally advanced angiocentric T cell lymphoma. Subsequently patient received 6-cycles of three weekly standard CHOP regimen with injection cyclophosphamide $750 \mathrm{mg}$ / 
$\mathrm{m}^{2}$, injection doxorubicin $50 \mathrm{mg} / \mathrm{m}^{2}$, injection vincristine $1.4 \mathrm{mg} /$ $\mathrm{m}^{2}$ and tablet prednisolone $50 \mathrm{mg} / \mathrm{m}^{2}$ day 1 to 5 . There was relief in local symptoms then she underwent PET-CT which revealed mucosal thickening in bilateral maxillary sinuses with complete obliteration of left maxillary sinus and dehiscent medial wall of right maxillary sinus, also erosion of anterior hard palate and involvement of left infraparotid and superficial cervical lymph nodes with increase uptake in bilateral thyroid lobes. For this the patient received high palliative external beam radiotherapy (EBRT) $35 \mathrm{~Gy} / 20 \# / 4$ weeks. Patient was on regular follow-up, and had residual midline hard palate defect of $4 \times 4 \mathrm{~m}$ with minimal necrotic debris and regular borders therefore in view of residual disease, she was planned for per oral lenalidomide chemotherapy $25 \mathrm{mg}$ once daily for 21 days followed by 1 week gap $\times 6$ courses. Patient is having considerable regression of localized disease and got superb palliation and is presently on drug holiday.

\section{Discussion}

Angiocentric nasal-type NK/T-cell lymphoma exhibits a distinctive geographical scattering, with predominance in Asia and Central and South America, whereas it is infrequent in Western populations. ${ }^{3}$ This idiosyncratic racial pattern may to some extent imply genetic factors, but is also strongly associated with high incidence of EBV infection. ${ }^{4-6}$ The median age of presentation is 52years, with a wide range of 3 to 94 years and a male-to-female ratio of $3: 1$. $^{7,8}$

Head and neck is the second most common region for the extranodal lymphomas after gastrointestinal tract. Approximately $2.5 \%$ of malignant lymphoma arises in Walleye's ring i.e., the tonsils, nasopharynx and base of the tongue. ${ }^{9}$ Extranodal nasal-type NK/Tcell lymphoma show predilection for upper aero digestive tracts, particularly nasal and paranasal areas ( $80 \%$ cases $) .3,4$

As in the present case report, typically the disease takes aggressive course, initially localized to the nasal region with complaints of mild to moderate intensity pain, non-specific rhinitis, sinusitis and nasal obstruction. As the disease progresses in advance stages, it presents as severe pain, epistaxis, facial swelling with swelling of the soft palate or posterior hard palate. ${ }^{10-12}$ With further progression, it may form a deep necrotic ulcer in the midline of palate destroying palatal tissues thus creating an oronasal fistula complicated with secondary infection having foul smelling discharge and halitosis and life-threatening hemorrhage. ${ }^{12}$ Bone marrow involvement occurs in less than $10 \%$ of patients and distant metastasis is unusual. ${ }^{13}$

Prognosis of patients is associated with numerous factors, including bone marrow infiltration, tumor invasion, existence of group B symptoms (fever $>38^{\circ} \mathrm{C}$ for $>3$ days, night sweats and weight loss of $>10 \%$ in six months), Epstein-Barr virus DNA level of circulating blood, granzyme B, CD94, Ki-67 expression and hemoglobin concentration prior to treatment. ${ }^{14}$ Clinically, the most commonly used risk assessment system with a proven prognosis value is the international prognostic index. ${ }^{15}$

Computerized tomography (CT) has been established to assess the extent of the disease as well distant metastases. Magnetic resonance imaging (MRI) has shown to better define local soft tissue and bony involvement. NK-cell lymphomas are FDG-avid and positron emission tomography computerized tomography (18-FDG PET-CT) offers more accurate definition of the magnitude of involvement therefore, used to establish the diagnosis and further for monitoring of treatment response. This has important implications in radiotherapy planning. PET, however, may not detect morphologically occult marrow infiltration uncovered by in-situ hybridization (ISH) for Epstein-Barr encoding region (EBER). ${ }^{16}$

Excisional biopsy or deep biopsy is often necessary for the diagnosis of this disease. The diagnosis is based on the histopathological appearance and immunohistochemical profile of the biopsy tissue and the analysis of T-cell receptor genes. ${ }^{17}$ The characteristic histopathological picture in NK/T cell lymphoma shows angiocentric and angiodestructive growth pattern with zonal necrosis. The neoplastic cells demonstrates the atypical lymphoid cells with T-cell phenotype and illustrates wide spread positivity for CD3, CD43, CD45RO, CD20 and CD57 immunocytochemical stain. Nasal variety of NK/T cell lymphoma reveal specific characteristics of natural killer (NK) cells. NK cells commonly appear as small lymphocytes with azurophilic granules and immunophenotypically express the characteristic CD56 marker. ${ }^{18}$ The tumor has a predominant EBV+ phenotype in about $85 \%$ of patients. ${ }^{19}$

Differential diagnosis can also be perplexing since symptoms such as secretion, nasal obstruction and ulceration may characterize other pathologies as well such as Wegener's granulomatosis (WG), blastomycosis, tuberculosis, adenocarcinoma, squamous cell carcinoma and nasal destruction for cocaine abuse. ${ }^{20}$

Currently, there is no standardized treatment protocol for ENKTCL. Patients with early stage (stage I and II) ENKTCL, radiotherapy alone retains high local relapse and distant tumor dissemination rates so these patients are mainly treated by combination of chemoradiation. ${ }^{21}$ Most patients with advanced disease (stage III and IV) tend to be treated with chemotherapy, such as CHOP (cyclophosphamide, doxorubicin, vincristine, prednisone) or third-generation anthracycline-containing regimens, but usually have poor response to treatment and die within several months. Several reports demonstrated successful treatment using hematopoietic stem cell transplantation (HSCT) for these diseases. Currently, HSCT is the only therapy expected to be curative in advanced cases. However, the results of transplant during relapse are poor, and further requires the development of more effective chemotherapeutic regimens for NK-cell neoplasms. ${ }^{22}$

Recently, the NK-cell tumor study group started a phase I trial of new combination chemotherapy named SMILE. The SMILE regimen consists of a steroid hormone, methotrexate, ifosfamide, L-asparaginase and etoposide and is a dose-finding study for methotrexate and etoposide..$^{22}$

The investigation into ENKTCL targeted therapy has just begun; there has been no targeted drug entering clinical trials, and bortezomib, a type of proteosome inhibitor, has demonstrated curative effects in certain preliminary studies. ${ }^{23}$ However, for advanced stage tumors, numerous types of treatments are of poor curative effect, and stronger or novel effective treatment programs require consideration.

\section{Conclusion}

Angiocentric T-cell lymphoma is a highly aggressive and rapidly progressively tumor with poor prognosis. The median survival time is $<12$ months and five-year survival rate is $30-40 \%$. Early-stage localized nasal disease is highly curable whereas disseminated disease has very poor prognosis. This underlines the importance of early diagnosis and therapy but the non-specific nature of early clinical signs and symptoms leads to diagnostic delay. Histopathological and immuno histo chemical evaluation of the lesion are mandatory for 
diagnosis. Combination of loco regional radiotherapy and CHOP chemotherapy are the mainstay of treatment till now. Additionally, autologous hematopoietic stem cell transplantation can be performed. Bortezomib has shown promising results in early disease but needs further research. Prosthetic treatment of possible or nasal fistulas/ defects can significantly improve the patient's quality of life. Present case highlights the important role of local radiotherapy and metronomic chemotherapy with lenalidomide for symptomatic improvement and further recommends more metronomic chemotherapeutic trials for such locally advanced patients.

\section{Acknowledgements}

None

\section{Conflict of interest}

Author declares that there is no conflict of interest.

\section{References}

1. Nikolaos N, Grigorios P, Konstantinos K, et al. Extranodal nasal-type NK/T-cell lymphoma of the palate and paranasal sinuses. Am J Case Rep. 2012;13:79-85

2. Wood PB, Parikh SR, Krause JR. Extranodal NK/T-cell lymphoma, nasal type. Proc (Bayl Univ Med Cent). 2011;24(3):251-254.

3. Au WY. Current management of nasal NK/T-cell lymphoma. Oncology (Williston Park, NY). 2010;24(4):352-358.

4. Suzuki R, Takeuchi K, Ohshima K, et al. Extranodal NK/T-cell lymphoma: diagnosis and treatment cues. Hematol Oncol. 2008;26(2):66-72.

5. Ohtsuka R, Abe Y, Sada E, et al. Adult patient with Epstein-Barr virus (EBV)-associated lymphoproliferative disorder: chronic active EBV infection or de novo extranodal natural killer (NK)/T-cell lymphoma, nasal type? Intern Med. 2009;48(6):471-474.

6. Arber DA, Weiss LM, Albujar PF, et al. Nasal lymphomas in Peru, high incidence of T-cell immunophenotype and Epstein-Barr virus infection. Am J Surg Pathol. 1993;17(4):392-399.

7. Wu X, Li P, Zhao J, et al. A clinical study of 115 patients with extranodal natural killer/T-cell lymphoma, nasal type. Clin Oncol (R Coll Radiol). 2008;20(8):619-625.

8. Meng W, Zhou Y, Zhang H, et al. Nasal-type NK/T-cell lymphoma with palatal ulcer as the earliest clinical manifestation: a case report with literature review. Pathol Oncol Res. 2010;16(1):133-137.

9. Essadi I, Ismaili N, Tazi E, et al. Primary lymphoma of the head and neck: two case reports and review of the literature. Cases Journal. 2008;1(1):426.
10. Rodrigo JP, Suárez C, Rinaldo A. Idiopathic midline destructive disease: fact or fiction. Oral Oncol. 2005;41(4):340-348.

11. Ketharanathan N, van Kipshagen PJ, Vasmel W, et al. T/NK cell lymphoma presenting as a "blocked nose". Eur Arch Otorhinolaryngol. 2008;265(9):1131-1134.

12. Huang KJ, Wang LF, Lee KW, et al. Sinonasal NK/T-cell lymphoma with upper gastrointestinal bleeding: a case report. Kaohsiung J Med Sci. 2003;19(12):639-643.

13. Gill H, Liang RHS, Tse E. Extranodal Natural-Killer/T-Cell Lymphoma, Nasal Type. Adv Hematol. 2010;2010:627401.

14. Jaccard A, Petit B, Girault S, et al. L-asparaginase-based treatment of 15 western patients with extranodal NK/T-cell lymphoma and leukemia and a review of the literature. Ann Oncol. 2009;20(1):110-116.

15. Chim CS, Ma SY, Au WY, et al. Primary nasal natural killer cell lymphoma: long-term treatment outcome and relationship with the International Prognostic Index. Blood. 2004;103(1):216-221.

16. Khong PL, Pang CBY, Liang R, et al. Fluorine-18 fluorodeoxyglucose positron emission tomography in mature $\mathrm{T}$-cell and natural killer cell malignancies. Ann Hematol Oncol. 2008;87(8):613-621.

17. Gourin CG, Johnson JT, Selvaggi K. Nasal T cell lymphoma: case report and review of diagnostic features. Ear Nose Throat J. 2001;80:458-460.

18. Tsang WM, Tong AC, Lam KY, et al. Nasal T/NK cell lymphoma: report of 3 cases involving the palate. J Oral Maxillofac Surg. 2000;58(11):1323-1327.

19. Schwartz EJ, Molina-Kirsch H, Zhao S, et al. Immunohistochemical Characterization of Nasal-Type Extranodal NK/T-Cell Lymphoma Using a Tissue Microarray. An Analysis of 84 Cases. Am J Clin Pathol. 2008;130(3):343-351.

20. Rodrigo JP, Suarez C, Rinaldo A, et al. Idiopathic midline destructive disease: fact or fiction. Oral Oncol. 2005;41(4):340-348.

21. Chiattone CS. Extranodal nasal type NK/T-cell lymphoma. Rev Bras de Hematol Hemoter (Impresso). 2009;31(2):26-29.

22. Metgud RS, Doshi JJ, Gaurkhede S, et al. Extranodal NK/T-cell lymphoma, nasal type (angiocentric T-cell lymphoma): A review about the terminology. J Oral Maxillofac Pathol. 2011;15(1):96-100.

23. Shen L, Au WY, Guo T, et al. Proteasome inhibitor bortezomib-induced apoptosis in natural killer (NK)-cell leukemia and lymphoma: an in vitro and in vivo preclinical evaluation. Blood. 2007;110:469-470. 